(C) 2016 IEEE. Personal use of this material is permitted. Permission from IEEE must be obtained for all other uses, in any current or future media, including reprinting/republishing this material for advertising or promotional purposes, creating new collective works, for resale or redistribution to servers or lists, or reuse of any copyrighted component of this work in other works. 


\title{
Control of Circulating Currents in Modular Multilevel Converters through Redundant
}

\section{Voltage Levels}

Georgios Konstantinou, Member, IEEE, Josep Pou, Senior Member, IEEE,

Salvador Ceballos, Ricard Picas, Student Member, IEEE,

Jordi Zaragoza, Member, IEEE, and Vassilios G. Agelidis, Senior Member, IEEE,

\begin{abstract}
Amongst the main control targets in a modular multilevel converter (MMC) is the control of the circulating currents within the phase-legs of the topology. This paper presents a controller for the circulating current of the MMC that utilises the available redundancies of the multilevel waveform in $2 N+1$ modulated MMCs in order to regulate the circulating current to its reference. The main advantages of the approach are the elimination of control loops that generate the reference voltages for the control of the circulating current, simple implementation and very fast dynamic performance. The controller is implemented at the modulation stage and its operation is independent of the circulating current reference. An extension of the controller to track large deviations in the circulating current is also demonstrated. The simplicity and effectiveness of the proposed controller is illustrated through detailed simulations and experimental results from a single-phase laboratory prototype.
\end{abstract}

\section{Index Terms}

Circulating current control, pulse-width modulation, multilevel converters, modular multilevel converter

\section{* Corresponding Author:}

Dr. Georgios Konstantinou,

Australian Energy Research Institute (AERI)

School of Electrical Engineering and Telecommunications

Tyree Energy Technologies Building, (Bldg H6)

University of New South Wales, Sydney, 2052, NSW, Australia

phone: +61 - 2 - 9385-7405 e-mail: g.konstantinou@unsw.edu.au 


\section{INTRODUCTION}

The family of multilevel converters based on series connection of sub-modules (SMs) [1], spearheaded by the modular multilevel converter (MMC, Fig. 1) [2]-[4] has defined the state of the art in multilevel power electronics conversion for high-power applications. Modular multilevel converters offer an expandable and redundant configuration [5] capable of generating a large number of voltage levels and providing high quality voltage and current waveforms with high efficiency and rduced switching losses [3], [4].

Owing to its multiple advantages and the provision of a single dc-link for the overall topology without requiring a dc-link capacitor, the MMC is well-suited to grid applications. Its most prominent use is in high-voltage direct-current (HVDC) power transmission while other applications include static synchronous compensators (STATCOMs), traction systems [3] and battery energy storage systems (BESS) [3], [4]. The converter has potential in the area of variable speed drives (VSD) [6], with the low-speed, low fundamental frequency operation and reduction of SM capacitor voltage ripple posing a particular set of challenges for the operation of the converter.

Major tasks for the control and proper operation of the MMC include the control of the output (voltages and currents) as well as internal (SM capacitor voltages and circulating currents) quantities. Regulating the SM capacitor voltages is imperative in order to provide balanced and symmetrical output while maintaining the ratings and limits of the SMs. On the other hand, circulating currents have a significant effect in the optimal operation of the converter while also affecting the ratings and power losses of the MMC. For both quantities, a set of controllers [7]-[25] is typically employed to ensure that all requirements and restrictions are met during the operation of the converter.

The objective of this paper is to propose and demonstrate the operation of a controller for the MMC circulating currents that utilises the pulse-width modulation patterns and, more specifically, the available redundancies in the voltage waveform of an MMC under $2 N+1$ modulation. The controller acts directly on the circulating current, selecting the number of the SMs within the arm so that the circulating current is driven to its reference value. It eliminates the need for additional voltage reference injections to the output voltage reference of the converter or further control loops.

The rest of the paper is organised in the following manner. Section II provides an overview of MMC circulating currents and relevant circulating current control methods proposed in the existing technical literature. Section III presents relevant background of $2 N+1$ modulation for the MMC and introduces the circulating current controller at the modulation stage based on the redundant switching states. Simulation and experimental results of the proposed controller are given in Sections IV and $\mathrm{V}$ and the conclusions of the work are summarised in Section VI. 


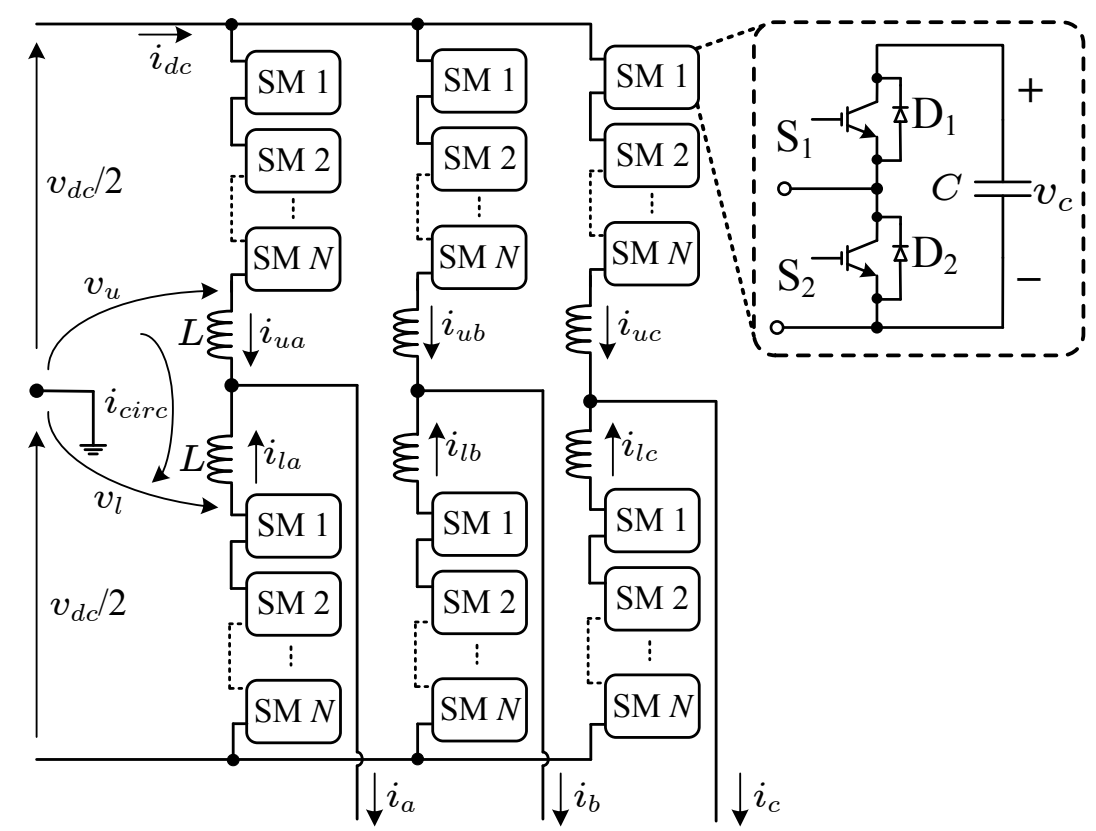

Fig. 1. Circuit configuration of a three-phase MMC.

\section{Circulating Current and Control in Modular Multilevel Converters}

Assuming an infinite number of SMs within the MMC arms $(N \rightarrow \infty)$, the converter can be represented with the equivalent circuit of Fig. 2(a). The value of the variable capacitors $C_{u}$ and $C_{l}$ depends on the number of series-connected SMs per arm. Under the assumption of $L \rightarrow 0$, the output current will be distributed amongst the two arms based on the value of these capacitors and, hence, the number of inserted SMs. Under a sinusoidal modulation signal $v_{a m}\left(v_{a m} \in[-1,1]\right)$

$$
v_{a m}=m_{a} \cos (\omega t)
$$

where $m_{a}$ represents the converter modulation index, and a phase current $i_{a}$ which is expressed as:

$$
i_{a}=\hat{I}_{a} \cos (\omega t+\phi),
$$

the currents through the upper and lower arms, and consequently through the upper and lower arm capacitors, can be written as [20]:

$$
i_{u}=\frac{\hat{I}_{a}}{2} \cos (\omega t+\phi)+\frac{m_{a} \hat{I}_{a}}{4} \cos (2 \omega t+\phi)+\frac{m_{a} \hat{I}_{a}}{4} \cos (\phi),
$$

and

$$
i_{l}=\frac{\hat{I}_{a}}{2} \cos (\omega t+\phi)-\frac{m_{a} \hat{I}_{a}}{4} \cos (2 \omega t+\phi)-\frac{m_{a} \hat{I}_{a}}{4} \cos (\phi) .
$$

The first term in (3) and (4) is equal to half of the phase current demonstrating that the upper and lower phase-arms share the output current $i_{a}$ equally. The second and the third terms in (3) and (4) represent the second order harmonic and the dc component of the circulating current within the 


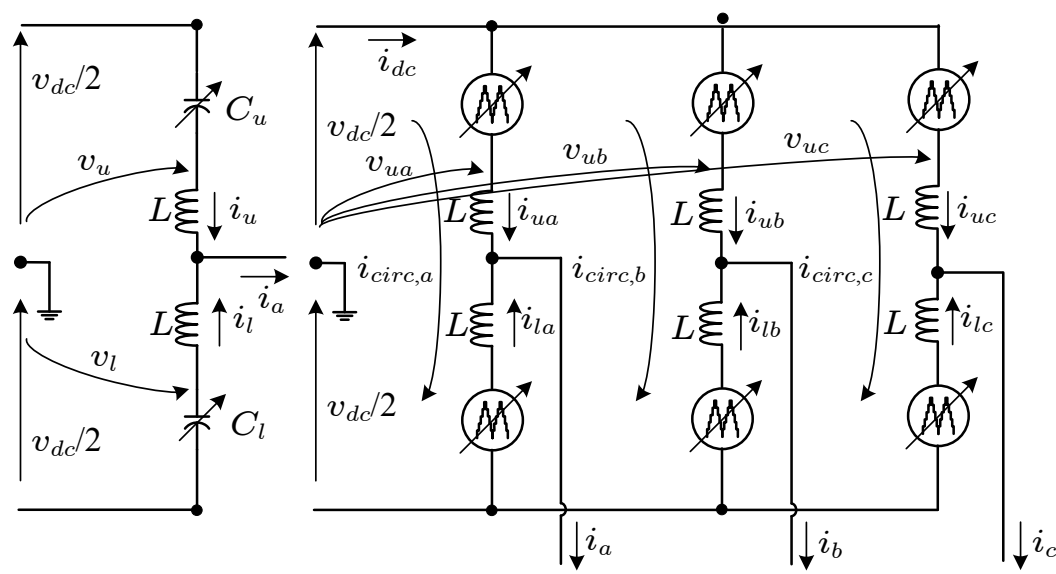

(a)

(b)

Fig. 2. Equivalent circuits of the MMC, (a) assuming infinite number of SMs in the converter arms, (b) three-phase circuit with converter arms as variable sources.

upper and lower phase-arms, respectively. Although in a practical MMC, the value of $L$ will not be equal to zero and the number of SMs, $N$, will not be infinite, the arm currents of (3) and (4) define the desired arm currents of the MMC [20].

An MMC may operate without an internal arm $\left(i_{u}\right.$ and $\left.i_{l}\right)$ or circulating current controller. However, such operation leads to an unregulated second order harmonic of (3) and (4). As it significantly compromises the efficiency of the converter and adds excessive stress to the semiconductor devices of the SMs, it is not practical to operate an MMC without some sort of circulating current controller. Additional benefits can be attained if the circulating current is regulated to a reference value which can be determined based on various control objectives. The benefits from controlling the circulating current have been identified from the very early stages of work in the topology [17], [21] and multiple controllers with different control objectives have been proposed in the existing literature [7]-[24].

In order to maintain the energy within the SM capacitors to the required level, a dc current component is necessary within the circulating current. From (3) and (4), the value of the dc current is calculated as:

$$
I_{d c}=\frac{m_{a} \hat{I}_{a}}{4} \cos \phi
$$

The dc value of (5) defines the simplest reference of the circulating current for the MMC $\left(i_{c i r c}=I_{d c}\right)$. To achieve the dc reference, the higher order harmonics and predominantly the negative sequence second order harmonic should be suppressed. This can be achieved in the synchronous reference frame (SRF) with the use of linear controllers implemented as a circulating current suppressing controller (CCSC) [21] or through directly filtering the higher order harmonics with the use of low-pass filters (LPF) in the measured circulating current [9]. An implementation of the latter is shown in Fig. 3 (a). A direct calculation of the reference to the value of (5) was shown to improve the dynamic 
performance of the controller [9] under transient conditions with such methods also applicable to model predictive control of the converter [8] and to MMCs operating under unbalanced conditions [11]. A circulating current control method based on the energy within the arms and controllers on the positive and negative synchronous reference frame was presented in [7].

As mentioned earlier, the predominant harmonic that needs to be eliminated from the circulating current is of second order, meaning that proportional or proportional integral controllers might not offer the necessary tracking characteristics. In order to improve the performance of reference tracking, various control methods that include resonant [10], [14], [24] and repetitive [15], [16] controllers have also been proposed, with an example shown in Fig. 3(b). Resonant controllers can be included for higher frequencies (such as the 4th, 6th etc) [24] without affecting the dc component of the circulating current but every additional loop adds to the complexity of the control system. The impact of deadtime in the performance of PR controllers was presented in [10], where an increase in the \%THD and a deterioration in the system performance was identified as a result of increased dead-times. The operation of the MMC under both balanced and unbalanced conditions considering non-ideal PR controllers, in an effort to reduce the infinite gain of ideal PR controllers at the resonant frequency, was shown in [14].

Elimination of higher order harmonics in the circulating current provides the lowest rms value for the arm current, resulting in the most efficient operation of the converter in terms of switching and conduction losses [9]. However, including higher order harmonics in the circulating current [17]-[19] provides secondary benefits to the MMC, predominantly in reducing the SM capacitor voltage ripple [17], allowing for a reduction in the size of the SM capacitors and the energy stored within the MMC topology. The off-line optimisation of [18] calculates the optimal level of even-order harmonics (a second and a combination of a second and fourth order harmonics) in the circulating current for all operating points and load conditions leading to a minimum in the SM capacitor voltage ripple. Analytical methods of calculating these harmonics [17], [19] can provide similar performance. An issue of these methods is the high computational cost or complexity when more than two harmonics are included in the current reference and the overall increase in the rms value of the arm current.

In order to simplify the calculation of injection levels, a reference for the circulating current can be derived from instantaneous values readily available to the current controller [20] by calculating the instantaneous capacitance with the upper and the lower arms of the phase-leg as:

$$
\begin{gathered}
C_{u}=\frac{C}{n_{u}}, \text { and } \\
C_{l}=\frac{C}{n_{l}},
\end{gathered}
$$

where $n_{u}$ and $n_{l}$ s the number of connected SMs in the upper and lower arm of the MMC, respectively. Assuming a sinusoidal variation in the number of SMs in the two arms based on the reference signal 


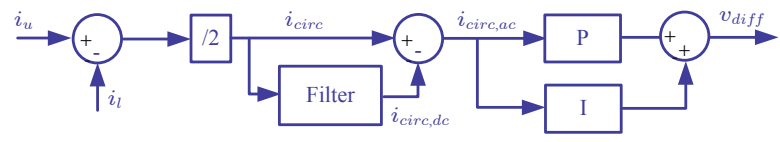

(a)

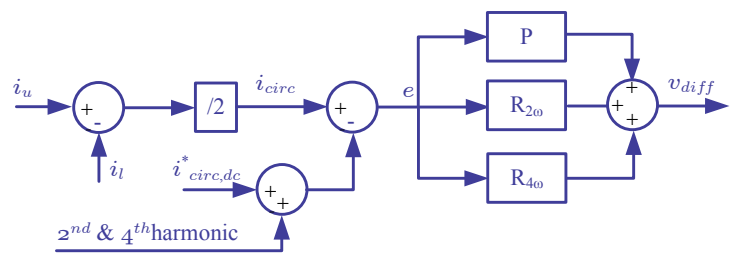

(b)

Fig. 3. Control of the circulating current, (a) elimination of ac components and, (b) injection of higher order harmonics

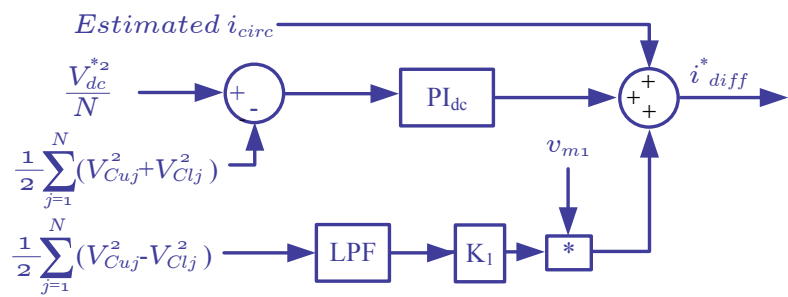

Fig. 4. Generation of circulating current control reference of the MMC using instantaneous measurement values.

of (1) the two arm currents can be written as

$$
\begin{aligned}
& i_{u}=i_{a} \frac{1+v_{a m}}{2}, \text { and } \\
& i_{l}=i_{a} \frac{1-v_{a m}}{2}, \text { and }
\end{aligned}
$$

and the reference for the circulating current can be calculated as

$$
i_{\text {circ }}=\frac{i_{a} v_{a m}}{2}
$$

with the total reference also including actions of PI controllers to facilitate energy balancing between the converter arms, as shown in Fig. 4. It was shown in [20] that the reference of (10) generates a circulating current similar to those of the analytically determined optimal injections in a computationally efficient manner and without calculation of the output current amplitude or phase angle.

A common point of all previous methods is that they use some sort of controller (PI, PR, repetitive, etc) in order to generate a voltage reference $\left(v_{d i f f}^{*}\right)$ for the control of the circulating current within each phase-leg. The voltage reference is then added and subtracted from the phase-leg reference $\left(v_{a m}\right)$ to generate the individual references of the two arms, providing control of the circulating current without affecting the output of the MMC phase-leg. 
An alternative approach uses the state-space model of the MMC [23] in order to provide an integrated strategy for the control of circulating currents and energy within the converter. Ref. [22] proposes the use of voltage correcting modules (VCMs), full-bridge SMs with lower voltage ratings and capable of generating positive and negative voltage at their terminals, in each of the arms of the MMC. The sole task of the VCMs is to provide control of the circulating current using only $v_{d i f f}^{*}$ as their reference.

\section{PRoposed IMPLEMENTATION}

This section introduces concepts of the $2 N+1$ modulation relevant to the description of the circulating current controller and develops the stages of the proposed controller. An extended form of the proposed controller is also presented.

\section{A. $2 N+1$ Modulation for the $M M C$}

A property of the MMC is that, for a given configuration with $N$ SMs per arm, it can generate $N+1$ or $2 N+1$ voltage levels in the output. This can be achieved either through carrier interleaving phase-shifting the carrier waveforms of the upper and lower arm by $180^{\circ}$ [26]-[28] - or by directly modulating the converter as a $(2 N+1)$-level (e.g. using $2 N$ carriers) and properly distributing the switching signals to the upper and lower arm SMs [29]. In both cases, if a sorting algorithm is used to facilitate voltage balancing, the only necessary output of the modulation stage is $n_{u}$ and $n_{l}$.

The additional voltage levels are intermediate levels to that of $N+1$ modulation (denoted as $x \pm 1 / 2$ in Fig. 5). These levels are generated when the number of SMs connected to the phase-leg of the converter is equal to either $N+1$ or $N-1$ [26] so that for level $x+1 / 2$ two possible combinations exist, one with $n_{l}=x$ and $n_{u}=N-(x+1)$ and a second with $n_{l}=x+1$ and $n_{u}=N-x$. These redundant states ( $R$ States of Fig. 7) are a result of connecting or removing SMs from either the upper or the lower arm so that $\left(N-n_{u}-n_{l}\right)$ is equal to \pm 1 . Fig. 5 shows the output voltage under $2 N+1$ modulation and the two possible combinations of the arm voltage waveforms assuming that one SM is either removed (middle figure) or added (lower figure) to total number SMs in the phase-leg.

Despite generating the same voltage level, the two redundant states have opposite effect in the circulating current. Considering the differential mode equivalent circuit of the MMC phase-leg (Fig. 6), the two states can be defined as the voltage of an additional SM $\left(v_{c}\right)$ with two different polarities, negative when $N+1$ and positive when $N-1$ SMs are connected in the phase-leg, as shown in Figs. 6(a) and (b) respectively. 


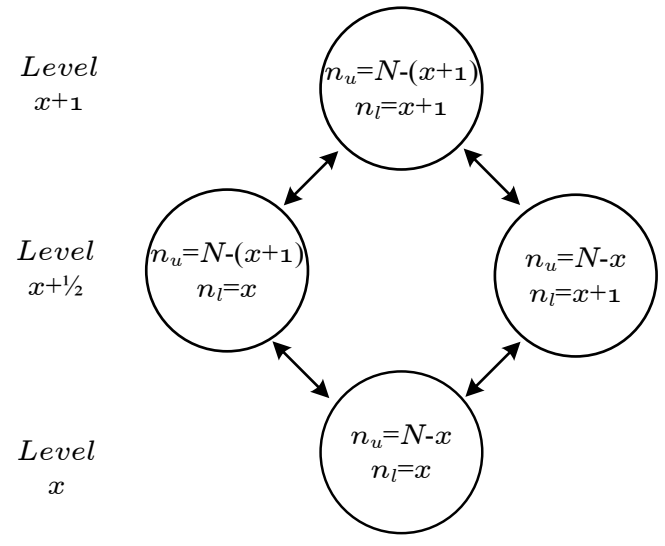

(a)

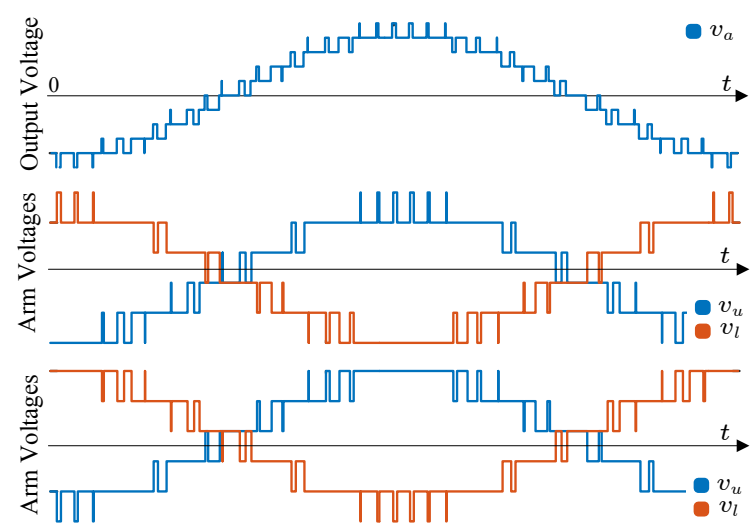

(b)

Fig. 5. 2N+1 modulation (a) Transitions between adjacent levels and number of SMs, and (b) Voltage waveforms (top figure: Thevenin equivalent voltage, middle figure: $N-1 \mathrm{SMs}$, bottom figure: $N+1 \mathrm{SMs}$ ).

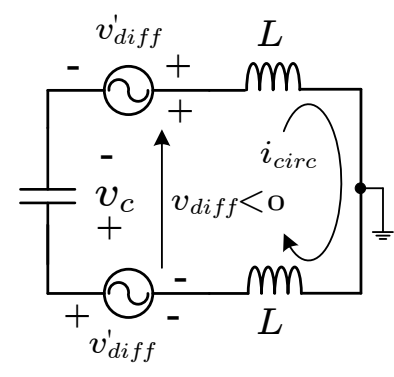

(a)

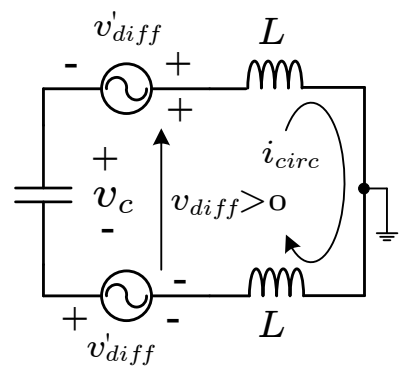

(b)

Fig. 6. Differential mode equivalent circuit. (a) $N+1 \mathrm{SMs}$ in the phase-leg, and (b) $N-1 \mathrm{SMs}$ in the phase-leg.

\section{B. Utilisation of Redundancies}

Based on the analysis of the previous section and the description of states from Fig. 5(b), we can construct a state diagram that demonstrates all possible voltage levels and their effect on the circulating current. Fig. 7 shows the diagram for five consecutive voltage levels of a generic multilevel waveform under $2 N+1$ modulation, which can be expanded to any MMC considering that the top and bottom levels are always non-redundant ones.

The proposed controller makes use of the two redundant states available, selecting the one that regulates the circulating current towards the reference. This means that when $i_{c i r c}$ is above the reference $\left(i_{\text {circ }}^{*}\right)$, then $N+1$ SMs will be connected to the phase leg and when $i_{\text {circ }}$ is below the reference, $N$-1 SMs will be connected. The only information, therefore, needed to select among the two states is whether $i_{\text {circ }} \geq i_{\text {circ }}^{*}$ or not. The above selection takes place during a transition from an $N R$ state to an $R$ state (Fig. 7) and can be treated as the connection or disconnection of one SM during the given transition. The reference signals for the upper and lower arm $\left(v_{a m u}\right.$ and $\left.v_{a m l}\right)$ remain 


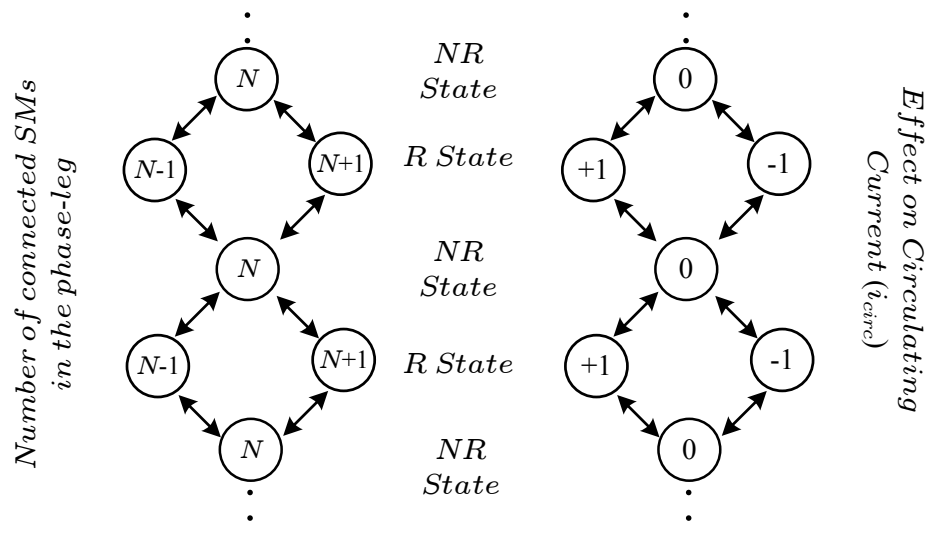

Fig. 7. Redundant switching states amongst consecutive voltage levels and effect on the circulating current.

unaffected. It should also be noted that the waveform will always transition to a non-redundant state after a redundant state (Fig. 7) so that no additional transitions will occur because of the proposed implementation, with the average switching frequency remaining constant.

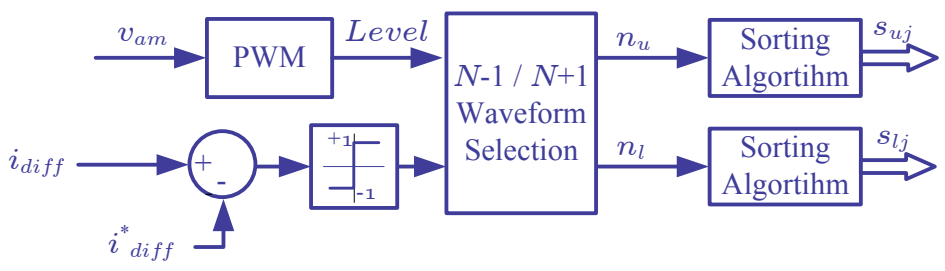

(a)

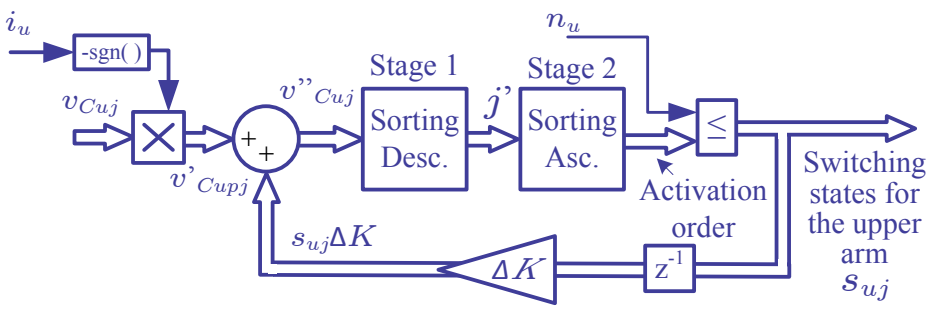

(b)

Fig. 8. MMC circulating current control and modulation. (a) Proposed implementation of circulating current control utilising a single redundancy, and (b) modified voltage sorting and balancing algorithm (upper arm).

The implementation of the proposed current controller is shown in Fig. 8(a). The reference signal together with the modulator define the level of the multilevel waveform, meaning that any of the existing PWM techniques can be readily applied. A simple comparison between the actual and the measured reference current provides the information necessary for the controller to select between the two redundant states. This also means that any of the control methods of Section II can be used as the reference $i_{\text {circ }}^{*}$. The output of the proposed controller is $n_{u}$ and $n_{l}$. 
Voltage balancing with reduced switching frequency of the converter is achieved through a restricted voltage balancing algorithm, as shown in Fig. 8(b). The algorithm uses two indices of the two sorting stages, in combination with the arm current direction and a feedback loop of the SM switching state, in order to derive the activation order of each SM within the arm. The virtual voltages $v_{c j}^{\prime \prime}$ used in the sorting stages of the algorithm are given by

$$
v_{c j}^{\prime \prime}=\operatorname{sgn}\left(i_{u}\right) \cdot v_{c j}+s_{u j} \Delta K \text {. }
$$

The feedback of the switching states together with an appropriate selection of the voltage separation $\Delta K$ ensures that only the requested number of SMs (typically only one) will change state maintaining the average switching frequency of the converter; see [25] for a detailed description of the voltage balancing algorithm.

\section{Extension of the concept}

The proposed controller is capable of selecting the necessary states to regulate the circulating current towards the selected reference. However, the action of the controller depends on the duration of the applied redundant state and also the voltage level of the SMs, as it is the voltage of one SM that is used to regulate the circulating current.

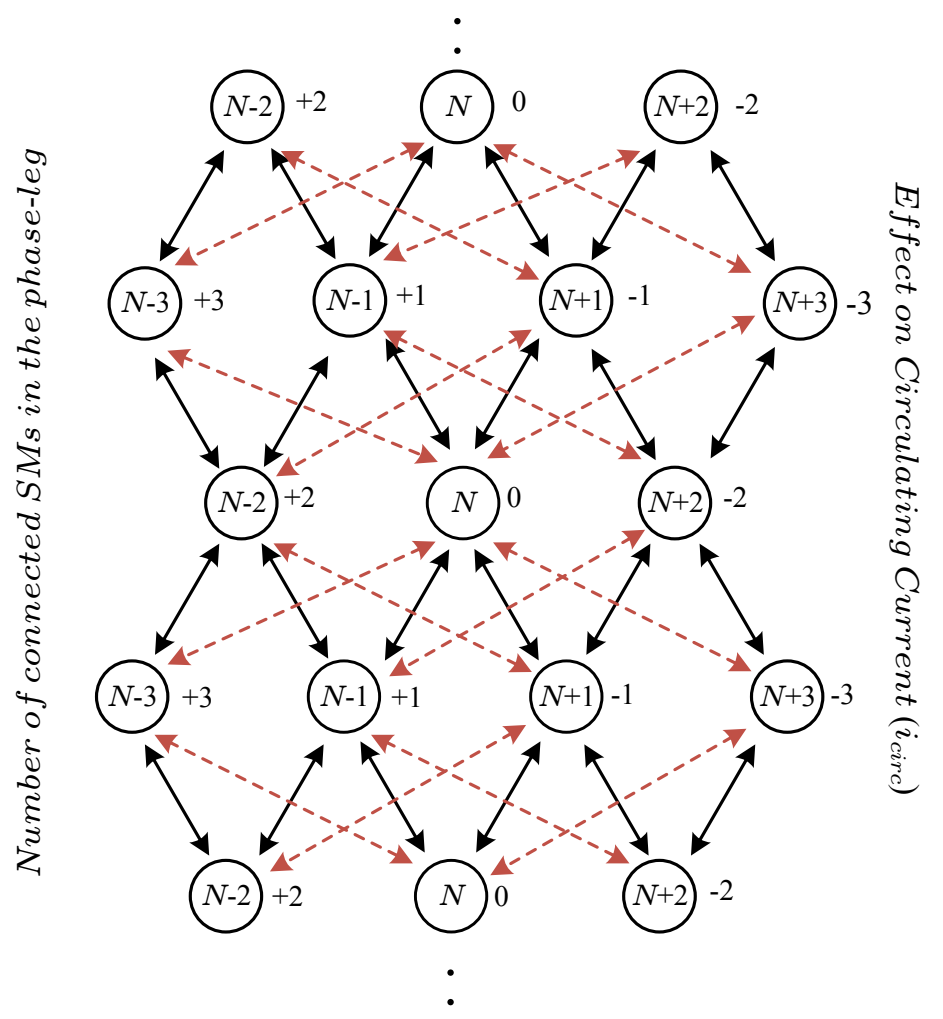

Fig. 9. Switching states and transitions within the phase-leg of the MMC demonstrating the extended implementation of the proposed circulating current controller. 
In cases where the deviation of the circulating current from its reference is particularly large, or when the change in the circulating current caused by the addition or subtraction of one SMs is not sufficient, then the concept of the proposed controller can be further extended to include additional "layers" of transitions amongst the SMs. Fig. 9 demonstrates the switching states of such an expansion with one additional layer. The additional states are available for all levels of the converter, with the exception of the top and the bottom level [30] and not limited to the $R$ states of Fig. 7. They introduce a deviation of two or three SMs in the $\left(N-n_{u}-n_{l}\right)$ while the output voltage of the phase-leg remains unaffected.

The extension of the concept creates two distinct groups of transitions, as demonstrated in Fig. 9. Transitions between adjacent states (indicated in black solid lines) only require one SM to change state and do not affect the SM switching frequency. However, transitions between levels which are further apart (red dashed lines) require multiple SM to connect and disconnect from both arms of the MMC. These transitions affect the overall switching frequency and are typically avoided as transitions between adjacent levels are typically preferred. An implementation of the extended concept is shown in Fig. 10 where the additional transitions are added or subtracted from the required number of SMs $\left(n_{u}\right.$ and $\left.n_{l}\right)$. The limiter is included in the path in order to avoid distortions to the output voltage and it functions similarly to a block that prevents overmodulation of the converter.

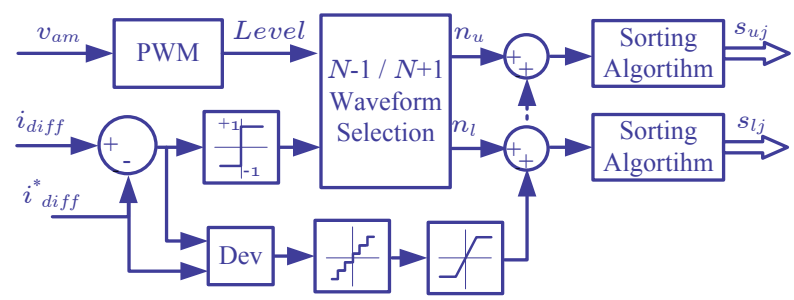

Fig. 10. Extended implementation for larger numbers of SMs.

\section{Simulation Results}

The operation of the proposed controller is demonstrated through simulations from an MMC with $N=50 \mathrm{SMs}$, capable of generating an 101-level waveform in the output voltage. The specifications of the system are given in Table I.

In order to demonstrate the proposed implementation, two different circulating current references are used for the control of the current, including the dc current reference of (5) and the dc plus ac reference of (10) [20]. The large number of levels and the number of available redundancies means that the extended implementation of Fig. 10 can be fully utilised.

Firstly, considering the dc reference of (5), the actual circulating current through the phase-leg of the converter as well as the output current are shown in Fig. 11. Due to the large number of 
TABLE I

Parameters of Simulation And Experimental Prototype

\begin{tabular}{ccc}
\hline \hline Parameter & Simulation & Experiment \\
\hline \hline Number of SMs per arm, $N$ & 50 & 5 \\
dc-link voltage, $V_{d c}$ & $100 \mathrm{kV}$ & $250 \mathrm{~V}$ \\
SM reference voltage, $V_{C}$ & $2 \mathrm{kV}$ & $50 \mathrm{~V}$ \\
SM capacitor, $C$ & $20 \mathrm{mF}$ & $3.6 \mathrm{mF}$ \\
Arm inductors, $L$ & $15 \mathrm{mH}$ & $3.6 \mathrm{mH}$ \\
Load $R_{L} \& L_{L}$ & $20 \Omega \& 20 \mathrm{mH}$ & $15.6 \Omega \& 5 \mathrm{mH}$ \\
Carrier frequency $f_{\text {car }}$ & $5 \mathrm{kHz}$ & $2 \mathrm{kHz}$ \\
\hline \hline
\end{tabular}

voltage levels, the output current demonstrates low harmonic distortion while the circulating current tracks the circulating current reference. The additional ripple that is present in the circulating current waveform is a result of the $2 N+1$ modulation and would be present regardless of the use of the proposed controller or not [26]. The additional deviation required to control the circulating current to its dc current reference under steady-state is shown in Fig. 12.

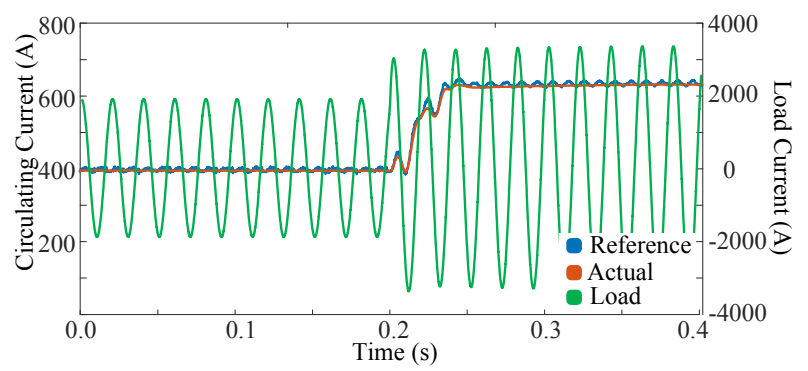

Fig. 11. Load Current, circulating current and circulating current reference under a step change in the load.

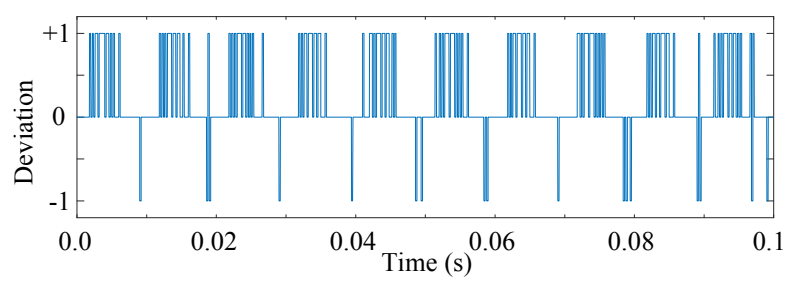

Fig. 12. Deviation added to the number of SMs in the upper and lower arm of the MMC in the extended implementation.

The operation and performance of the proposed controller can also be analysed from the perspective of harmonics in the circulating current. When a current controller is not implemented, the harmonic content of the circulating current is only influenced from the modulation of the upper and the lower 


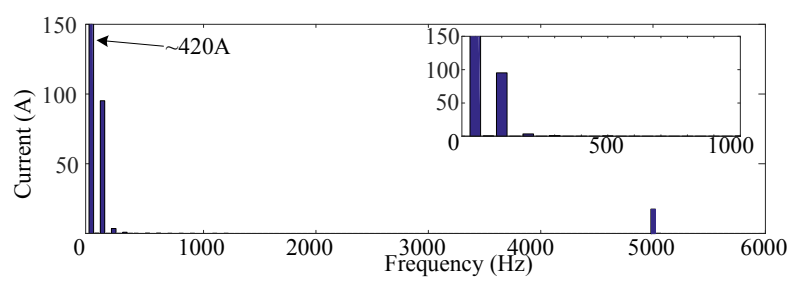

(a)

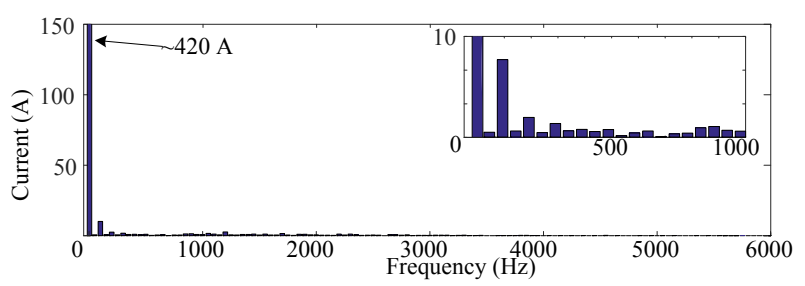

(b)

Fig. 13. Harmonic spectra of the circulating current under $2 N+1$ modulation (a) without current control, (b) with the proposed implementation.

arm, as shown in Fig. 13(a). Additionally, if $2 N+1$ modulation is used, a significant harmonic at the equivalent switching frequency of the converter [26] will also be present in the circulating current together with the second order harmonic. This harmonic is the switching frequency component of the upper and lower arms that is not canceled out in the circulating current due to the interleaved operation of the converter arms. The implementation of the proposed circulating current controller spreads the harmonic content of $i_{c i r c}$ as it tries to regulate it towards its reference, affecting the regular pattern and the higher order harmonics. This can be demonstrated through an FFT of the circulating current when the proposed implementation is used, as shown in Fig. 13(b) and the emergence of lower order harmonics (other than the second order) in the circulating current compared to the case without control.

To further demonstrate the performance of the proposed controller, a transition between the two circulating current references, from a dc circulating current to a dc plus ac one, is introduced in Fig. 14. The controller is capable of following the reference and tracking the transition almost instantaneously. The arm currents of the converter $\left(i_{u}\right.$ and $\left.i_{l}\right)$ are also given in Fig. 14(b). The SM capacitor voltages are well regulated to the reference value with Fig. 15 illustrating the capacitor voltages under the dc current reference.

\section{EXPERIMENTAL RESUlts}

The proposed algorithm utilising the single redundancy, based on Fig. 8(a), is implemented in a dSPACE DS1103 and applied to a single-phase MMC prototype with $N=5$ SMs per arm, as shown in Fig. 16(a). A photo of the full experimental setup and its parameters are given in Fig. 16(b) and 


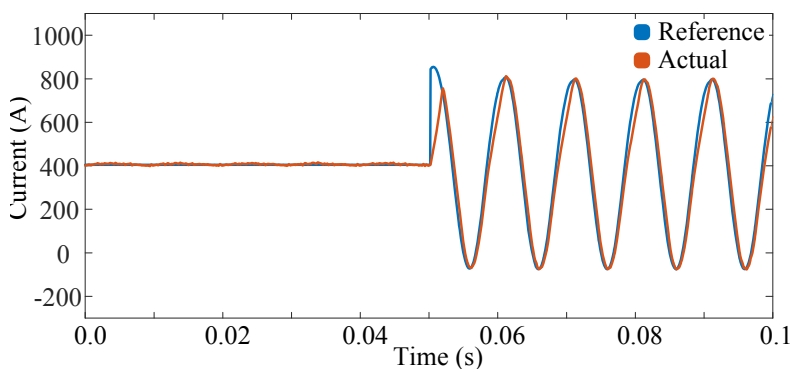

(a)

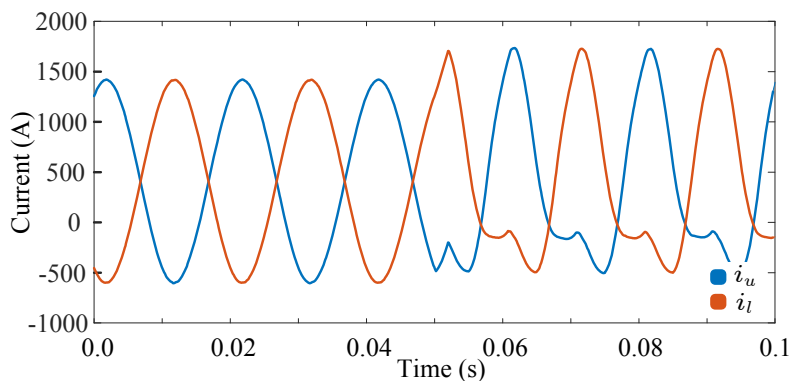

(b)

Fig. 14. Transition between the two circulating current references. (a) Circulating current, and (b) arm currents.

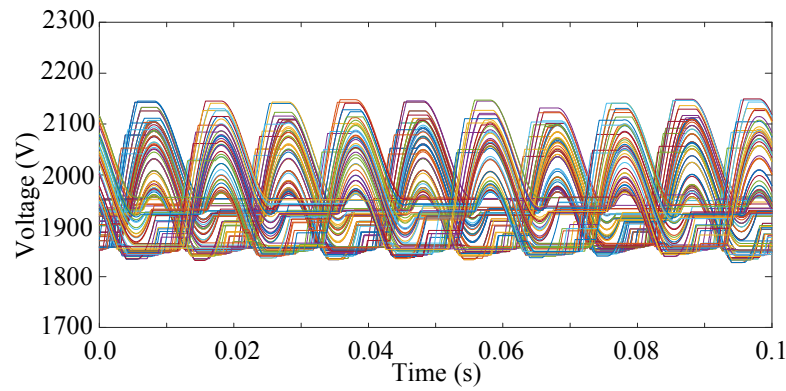

(a)

Fig. 15. Simulated SM capacitor voltages with the proposed circulating current control implementatio under a dc circulating current (100 traces).

Table I, respectively. The two circulating current references of (5) and (10) are both used in the experiment for the control of the circulating current and in order to demonstrate the performance of the proposed circulating current control implementation.

Figs. 17(a) and (b) show simulation and experimental results of the output voltage of the converter as well as the load and arm currents over ten periods of operation. At $t=0.06$, a transition between the two circulating current references is applied. As the converter with $N=5 \mathrm{SMs}$ is modulated under the $2 N+1$ scheme, the output voltage exhibits eleven voltage levels with low harmonic distortion. The arm currents consist of half the load current, the circulating current and the high order harmonics due to the modulation and the circulating current control (Fig. 13), which do not appear in the load current. 


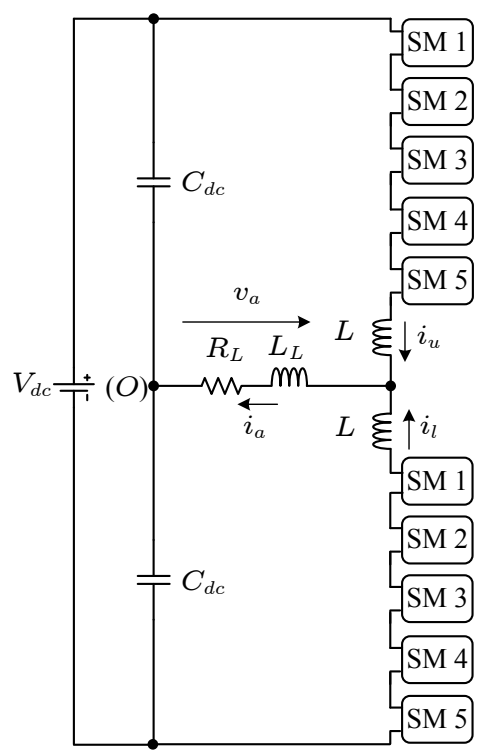

(a)

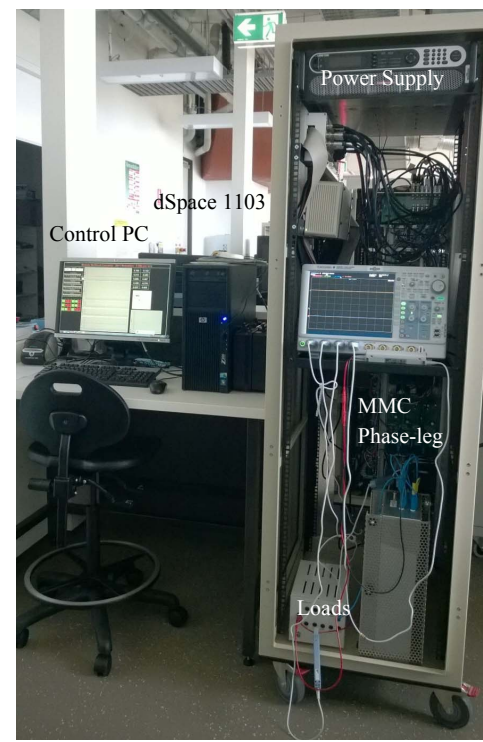

(b)

Fig. 16. Eleven-level experimental prototype converter. (a) Circuit diagram, and (b) laboratory prototype.

The simulation results of Fig. 17(a) and experimental results of Fig. 17(b) are in good agreement, verifying the proposed implementation.

The load $\left(i_{a}\right)$ and circulating currents $\left(i_{c i r c}\right)$ under the two different circulating current references are shown in Fig. 18(a) and (b). The effect of the circulating current control in the SM capacitor voltages is shown in Fig. 19 for the two circulating current methods with the ac circulating current reference of (10) also leading to a reduction in the SM capacitor voltage ripple. The overall operation of the converter remains unaffected, and transitions between the states only take place during changes in the voltage levels meaning that the switching frequency of the individual SMs is maintained low. Further extension of the control to include more than one level of redundancy cannot be experimentally demonstrated due to the low number of SMs in the prototype and the effective operation of the proposed controller.

\section{CONCLUSION}

Control of the circulating current within the MMC legs is usually achieved by modifying the upper and lower arm reference signals in order to follow the required current reference. This paper demonstrates that a similar task can be achieved by utilising the redundant voltage states of the MMC under $2 N+1$ modulation and selecting the number of SMs in each arm based on the deviation of the circulating current from its reference. The proposed method features a simple implementation and does not require complicated controllers that are necessary to accurately track the different circulating current references of the converter. Although initially based on the redundant states that are present 

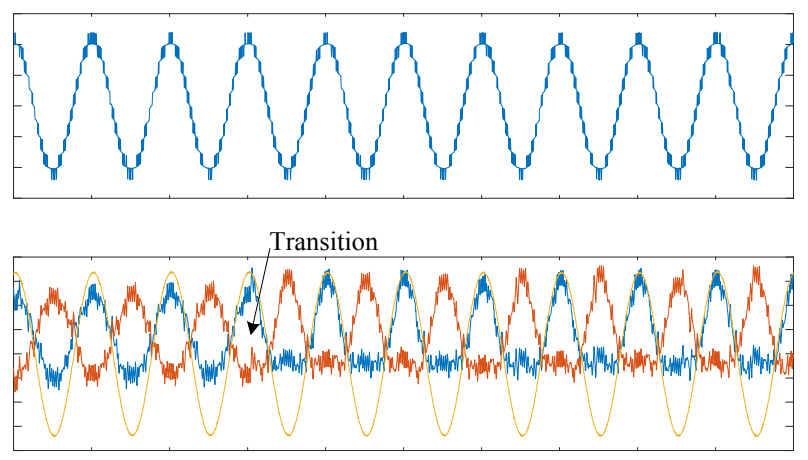

(a)

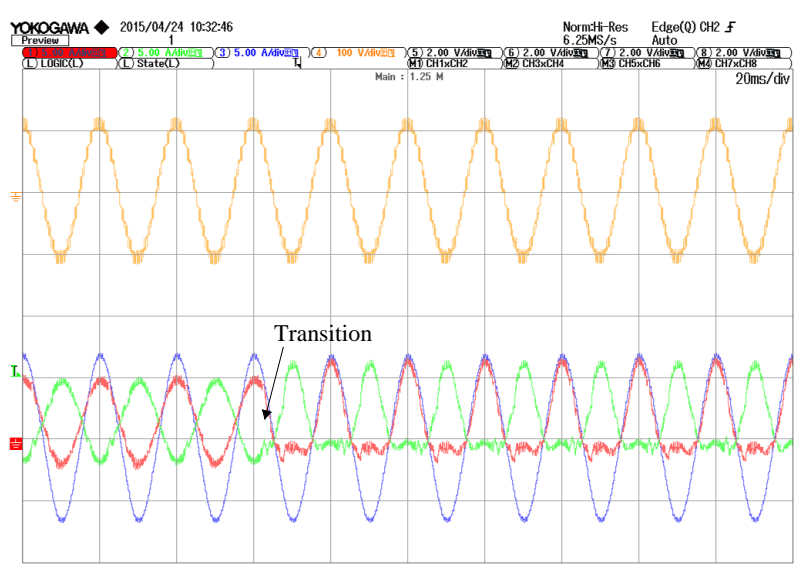

(b)

Fig. 17. Transition from the dc to the dc plus ac circulating current reference. (a) Simulation, and (b) Experimental Results; $\mathrm{CH} 1$ : upper arm current $i_{u}$; $\mathrm{CH} 2$ : lower arm current $i_{l}, \mathrm{CH} 3$ : load current $i_{a}$; $\mathrm{CH} 4$ : load voltage $v_{a}$; $\mathrm{CH} 1, \mathrm{CH} 2, \mathrm{CH} 3$ : 5A/div, CH4: 100V/div.

due to modulation, the method can be extended to make use of additional redundancies in order to regulate larger deviations of the circulating current from its reference, which can be particularly useful in converters with large numbers of SMs. The performance and effectiveness of the control method in regulating the circulating current both to dc and dc plus ac references is supported with results from a simulated system with $50 \mathrm{SMs}$ per arm and experimental results from a single-phase laboratory prototype with $5 \mathrm{SMs}$ per arm.

\section{REFERENCES}

[1] H. Akagi, "Classification, terminology, and application of the modular multilevel cascade converter (mmcc)," IEEE Trans. Power Electron., vol. 26, no. 11, pp. 3119-3130, Nov 2011.

[2] A. Lesnicar and R. Marquardt, "An innovative modular multilevel converter topology suitable for a wide power range," in Power Tech Conference Proceedings, 2003 IEEE Bologna, vol. 3, June 2003, pp. 6 pp. Vol.3-.

[3] M. Perez, S. Bernet, J. Rodriguez, S. Kouro, and R. Lizana, "Circuit topologies, modeling, control schemes, and applications of modular multilevel converters," IEEE Trans. Power Electron., vol. 30, no. 1, pp. 4-17, Jan 2015. 


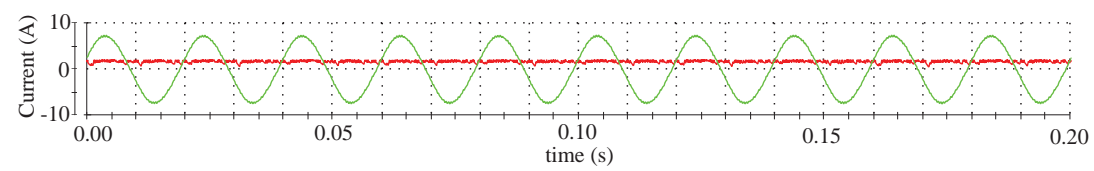

(a)

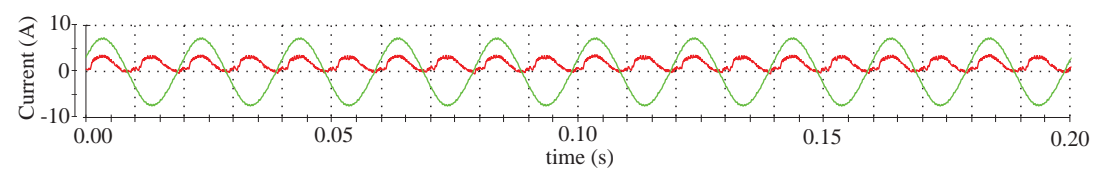

(b)

Fig. 18. Load and circulating currents of the single-phase MMC, (a) dc circulating current reference and (b) dc plus ac circulating current reference.

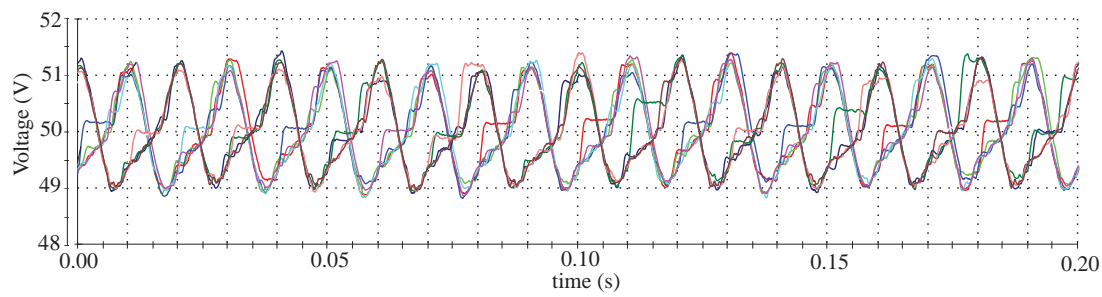

(a)

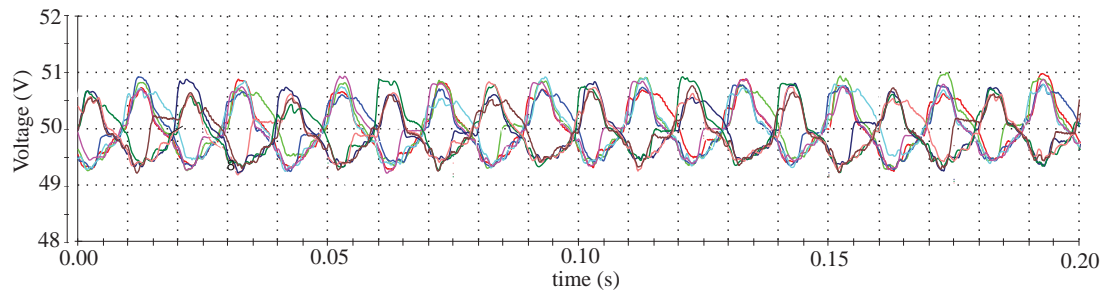

(b)

Fig. 19. SM capacitor voltages (10 traces per figure) with (a) a dc circulating current reference and (b) a dc plus ac circulating current reference.

[4] S. Debnath, J. Qin, B. Bahrani, M. Saeedifard, and P. Barbosa, "Operation, control, and applications of the modular multilevel converter: A review," IEEE Trans. Power Electron., vol. 30, no. 1, pp. 37-53, Jan 2015.

[5] G. Konstantinou, J. Pou, S. Ceballos, and V. Agelidis, "Active redundant submodule configuration in modular multilevel converters," IEEE Trans. Power Del., vol. 28, no. 4, pp. 2333-2341, Oct 2013.

[6] A. Antonopoulos, L. Angquist, S. Norrga, K. Ilves, L. Harnefors, and H.-P. Nee, "Modular multilevel converter ac motor drives with constant torque from zero to nominal speed," IEEE Trans. Ind. Appl., vol. 50, no. 3, pp. 1982-1993, May 2014.

[7] G. Bergna, E. Berne, P. Egrot, P. Lefranc, A. Arzande, J.-C. Vannier, and M. Molinas, "An energy-based controller for hvdc modular multilevel converter in decoupled double synchronous reference frame for voltage oscillation reduction," IEEE Trans. Ind. Electron., vol. 60, no. 6, pp. 2360-2371, June 2013.

[8] J. Bocker, B. Freudenberg, A. The, and S. Dieckerhoff, "Experimental comparison of model predictive control and 
cascaded control of the modular multilevel converter," IEEE Trans. Power Electron., vol. 30, no. 1, pp. 422-430, Jan 2015.

[9] R. Darus, J. Pou, G. Konstantinou, S. Ceballos, and V. Agelidis, "Circulating current control and evaluation of carrier dispositions in modular multilevel converters," in ECCE Asia Downunder (ECCE Asia), 2013 IEEE, June 2013, pp. $332-338$.

[10] B. Chen, Y. Chen, C. Tian, J. Yuan, and X. Yao, "Analysis and suppression of circulating harmonic currents in a modular multilevel converter considering the impact of dead time," IEEE Trans. Power Electron., vol. 30, no. 7, pp. 3542-3552, July 2015.

[11] J.-W. Moon, C.-S. Kim, J.-W. Park, D.-W. Kang, and J.-M. Kim, "Circulating current control in mmc under the unbalanced voltage," IEEE Trans. Power Del., vol. 28, no. 3, pp. 1952-1959, July 2013.

[12] D. Jovcic and A. Jamshidifar, "Phasor model of modular multilevel converter with circulating current suppression control," IEEE Trans. Power Del., vol. PP, no. 99, pp. 1-1, 2014.

[13] J. Jung, H. Lee, and S. Sul, "Control strategy for improved dynamic performance of variable-speed drives with modular multilevel converter," Emerging and Selected Topics in Power Electronics, IEEE Journal of, vol. 3, no. 2, pp. 371-380, June 2015 .

[14] S. Li, X. Wang, Z. Yao, T. Li, and Z. Peng, "Circulating current suppressing strategy for mmc-hvdc based on nonideal proportional resonant controllers under unbalanced grid conditions," IEEE Trans. Power Electron., vol. 30, no. 1, pp. 387-397, Jan 2015.

[15] L. He, K. Zhang, J. Xiong, and S. Fan, "A repetitive control scheme for harmonic suppression of circulating current in modular multilevel converters," IEEE Trans. Power Electron., vol. 30, no. 1, pp. 471-481, Jan 2015.

[16] M. Zhang, L. Huang, W. Yao, and Z. Lu, "Circulating harmonic current elimination of a cps-pwm-based modular multilevel converter with a plug-in repetitive controller," IEEE Trans. Power Electron., vol. 29, no. 4, pp. 2083-2097, April 2014.

[17] S. Engel and R. De Doncker, "Control of the modular multi-level converter for minimized cell capacitance," in Power Electronics and Applications (EPE 2011), Proceedings of the 2011-14th European Conference on, Aug 2011, pp. $1-10$.

[18] R. Picas, J. Pou, S. Ceballos, J. Zaragoza, G. Konstantinou, and V. Agelidis, "Optimal injection of harmonics in circulating currents of modular multilevel converters for capacitor voltage ripple minimization," in ECCE Asia Downunder (ECCE Asia), 2013 IEEE, June 2013, pp. 318-324.

[19] G. Maragliano, M. Marchesoni, and L. Vaccaro, "Optimal operation mode for modular multilevel converter based hvdc," in Power Electronics, Electrical Drives, Automation and Motion (SPEEDAM), 2014 International Symposium on, June 2014, pp. 784-789.

[20] J. Pou, S. Ceballos, G. Konstantinou, V. Agelidis, R. Picas, and J. Zaragoza, "Circulating current injection methods based on instantaneous information for the modular multilevel converter," IEEE Trans. Ind. Electron., vol. 62, no. 2, pp. 777-788, Feb 2015

[21] Q. Tu, Z. Xu, and L. Xu, "Reduced switching-frequency modulation and circulating current suppression for modular multilevel converters," IEEE Trans. Power Del., vol. 26, no. 3, pp. 2009-2017, July 2011.

[22] B. Riar and U. Madawala, "Decoupled control of modular multilevel converters using voltage correcting modules," IEEE Trans. Power Electron., vol. 30, no. 2, pp. 690-698, Feb 2015.

[23] P. Munch, D. Gorges, M. Izak, and S. Liu, "Integrated current control, energy control and energy balancing of modular multilevel converters," in IECON 2010 - 36th Annual Conference on IEEE Industrial Electronics Society, Nov 2010, pp. $150-155$.

[24] Z. Li, P. Wang, Z. Chu, H. Zhu, Y. Luo, and Y. Li, "An inner current suppressing method for modular multilevel converters," IEEE Trans. Power Electron., vol. 28, no. 11, pp. 4873-4879, Nov 2013. 
[25] R. Darus, J. Pou, G. Konstantinou, S. Ceballos, R. Picas, and V. Agelidis, “A modified voltage balancing algorithm for the modular multilevel converter: Evaluation for staircase and phase-disposition pwm," IEEE Trans. Power Electron., vol. 30, no. 8, pp. 4119-4127, Aug 2015.

[26] G. Konstantinou, M. Ciobotaru, and V. Agelidis, "Analysis of multi-carrier pwm methods for back-to-back hvdc systems based on modular multilevel converters," in IECON 2011 - 37th Annual Conference on IEEE Industrial Electronics Society, Nov 2011, pp. 4391-4396.

[27] K. Ilves, L. Harnefors, S. Norrga, and H.-P. Nee, "Analysis and operation of modular multilevel converters with phase-shifted carrier pwm,” IEEE Trans. Power Electron., vol. 30, no. 1, pp. 268-283, Jan 2015.

[28] B. Li, R. Yang, D. Xu, G. Wang, W. Wang, and D. Xu, "Analysis of the phase-shifted carrier modulation for modular multilevel converters," IEEE Trans. Power Electron., vol. 30, no. 1, pp. 297-310, Jan 2015.

[29] G. Konstantinou, M. Ciobotaru, and V. Agelidis, "Selective harmonic elimination pulse-width modulation of modular multilevel converters," IET Power Electron., vol. 6, no. 1, pp. 96-107, Jan 2013.

[30] G. Konstantinou, J. Pou, S. Ceballos, R. Darus, and V. Agelidis, "Switching frequency analysis of staircase modulated modular multilevel converters and equivalent pwm techniques," IEEE Trans. Power Del., vol. PP, no. 99, pp. 1-1, 2015. 